Article

\title{
PNPLA3 Expression Is Related to Liver Steatosis in Morbidly Obese Women with Non-Alcoholic Fatty Liver Disease
}

\author{
Gemma Aragonès ${ }^{1, \dagger}$, Teresa Auguet ${ }^{1,2, \dagger}$, Sandra Armengol ${ }^{1}$, Alba Berlanga ${ }^{1}$, \\ Esther Guiu-Jurado ${ }^{1}$, Carmen Aguilar ${ }^{1}$, Salomé Martínez ${ }^{3}$, Fátima Sabench ${ }^{4}$, \\ José Antonio Porras ${ }^{2}$, Maikel Daniel Ruiz ${ }^{2}$, Mercé Hernández ${ }^{4}$, Joan Josep Sirvent ${ }^{3}$, \\ Daniel Del Castillo ${ }^{4}$ and Cristóbal Richart ${ }^{1,2, *}$
}

1 Group de Recerca GEMMAIR (AGAUR)-Medicina Aplicada, Institut Investigació Sanitària Pere Virgili (IISPV), Departament de Medicina i Cirurgia, Universitat Rovira i Virgili (URV), 43007 Tarragona, Spain; gemma.aragones@iispv.cat (G.A.); tauguet.hj23.ics@gencat.cat (T.A.); sandra.armengol@urv.cat (S.A.); alba.berlanga@urv.cat (A.B.); esther.guiu@urv.cat (E.G.-J.); caguilar.hj23.ics@gencat.cat (C.A.)

2 Servei Medicina Interna, Hospital Universitari Joan XXIII Tarragona, Mallafré Guasch, 4, 43007 Tarragona, Spain; aporras.hj23.ics@gencat.cat (J.A.P.); drgorrin@yahoo.es (M.D.R.)

3 Servei Anatomia Patològica, Hospital Universitari Joan XXIII Tarragona, Mallafré Guasch, 4, 43007 Tarragona, Spain; mgonzalez.hj23.ics@gencat.cat (S.M.); jsirvent.hj23.ics@gencat.cat (J.J.S.)

4 Servei de Cirurgia, Hospital Sant Joan de Reus, Departament de Medicina i Cirurgia, Universitat Rovira i Virgili (URV), IISPV, Avinguda Doctor Josep Laporte, 2, 43204 Tarragona, Spain; fatima.sabench@urv.cat (F.S.); mhernandezg@grupsagessa.com (M.H.); ddelcastillo@grupsagessa.com (D.D.C.)

* Correspondence: crichart.hj23.ics@gencat.cat; Tel./Fax: +34-977-295-833

+ These authors contributed equally to this work.

Academic Editors: Giovanni Targher and Amedeo Lonardo

Received: 24 February 2016; Accepted: 22 April 2016; Published: 27 April 2016

\begin{abstract}
Recent reports suggest a role for the Patatin-like phospholipase domain-containing protein 3 (PNPLA3) in the pathology of non-alcoholic fatty liver disease (NAFLD). Lipid deposition in the liver seems to be a critical process in the pathogenesis of NAFLD. The aim of the present work was to evaluate the association between the liver PNPLA3 expression, key genes of lipid metabolism, and the presence of NAFLD in morbidly obese women. We used real-time polymerase chain reaction (PCR) analysis to analyze the hepatic expression of PNPLA3 and lipid metabolism-related genes in 55 morbidly obese subjects with normal liver histology (NL, $n=18)$, simple steatosis (SS, $n=20)$, and non-alcoholic steatohepatitis (NASH, $n=17$ ). Liver biopsies were collected during bariatric surgery. We observed that liver PNPLA3 expression was increased in NAFLD than in NL. It was also upregulated in SS than in NL. Interestingly, we found that the expression of PNPLA3 was significantly higher in severe than mild SS group. In addition, the expression of the transcription factors $L X R \alpha$, $P P A R \alpha$, and SREBP2 was positively correlated with PNPLA3 liver expression. Regarding rs738409 polymorphism, GG genotype was positive correlated with the presence of NASH. In conclusion, our results show that PNPLA3 could be related to lipid accumulation in liver, mainly in the development and progression of simple steatosis.
\end{abstract}

Keywords: PNPLA3; morbid obesity; non-alcoholic fatty liver disease; simple steatosis; fatty acid metabolism; non-alcoholic steatohepatitis

\section{Introduction}

Non-alcoholic fatty liver disease (NAFLD), the most common liver disease in Western countries, is characterized by the accumulation of excess triglycerides (TG) in hepatocytes and is associated 
with or anticipates the metabolic syndrome and its individual features, including visceral obesity, hyperlipidemia, and type 2 diabetes mellitus (T2DM) [1]. NAFLD includes a range of diseases from simple fatty infiltration (simple steatosis (SS)), fat accumulation, and inflammation (non-alcoholic steatohepatitis (NASH)) to liver fibrosis/cirrhosis [2]. General prevalence of NAFLD is $25.24 \%$, with the highest prevalence in the Middle East and South America. This prevalence is particularly high in obese adults $(80 \%-90 \%)$, patients with T2DM $(30 \%-50 \%)$, and up to $90 \%$ in patients with hyperlipidemia [3]. NAFLD is usually diagnosed by abdominal ultrasonography in subjects without any apparent liver alteration who do not consume excessive alcohol [4]. Some studies have shown that insulin resistance (IR) promotes not only the recruitment of free fatty acids (FAs) in liver from the serum pool, but also the accumulation of intrahepatic FA, which indicates that IR is, among other mechanisms, crucial to the pathogenesis of NAFLD/NASH. In this regard, some authors have attempted to explain the pathophysiology of NAFLD by advancing the "multiple parallel hits hypothesis" [5]. However, the specific process responsible for the development and progression of NAFLD is still an open question. While SS is considered a relatively benign condition with little risk of progression, NASH may progress to cirrhosis and, in a small percentage of patients, to hepatocellular carcinoma (HCC) [6]. In fact, there is increasing evidence to indicate a complex interplay between environmental genetic factors that predispose the progression of NAFLD [7].

Patatin-like phospholipase domain-containing protein 3 (PNPLA3), which is also known as adiponutrin, is mainly expressed in hepatocytes but also in adipocytes [8]. The protein is one of the candidates potentially related to NAFLD susceptibility. Regarding PNPLA3 lipase activity against TG and acylglycerol transacetylase activity, its expression is responsible for energy mobilization and the storage in lipid droplets $[9,10]$. Additionally, it is highly modulated by nutritional stimuli at transcriptional and posttranscriptional levels [11].

In 2008, Romeo et al. [12] reported that a PNPLA3 single nucleotide polymorphism at residue 148 in the DNA sequence, resulting in a substitution of isoleucine for methionine (I148M, rs738409), was a genetic determinant of NAFLD. Since then, the correlation between the PNPLA3 148M variant and NAFLD has been investigated in considerable detail. Multiple studies have demonstrated a link between the PNPLA3 148M variant and the development and progression of NAFLD, including liver fibrosis [13-18]. Recently, it has been reported that PNPLA3 148M elevates retinyl-palmitate content in human hepatic stellate cells providing evidence for a potential link between the PNPLA3 variant, human hepatic retinoid metabolism, and chronic liver disease [19,20]. All this research indicates that this variant is a potential modifier of NAFLD. Nevertheless, its role in the NAFLD development and the specific molecular mechanisms has not been fully elucidated.

Lipid deposition in the liver seems to be a critical mechanism in the pathogenesis of NAFLD, so its regulatory processes need to be elucidated if the progression of NAFLD is to be controlled. Although these potential regulatory mechanisms are multiple, one of them affecting TG remodeling could be PNPLA3 [21-23].

On the basis of this data, the aim of our work was to study the relationship between the liver expression of PNPLA3 and the presence of NAFLD in morbidly obese women. Furthermore, as lipid metabolism seems to be involved in the pathogenesis of NAFLD, we investigated the association between the hepatic expression of PNPLA3 and the expression of the main lipid metabolism-related genes. Finally, in order to explore the impact of the PNPLA3 genetic variant on the presence of NAFLD, we determined the relationships between the rs738409 polymorphism in the PNPLA3 gene and the severity of the disease.

\section{Results}

\subsection{General Characteristics of Cohort}

Our morbidly obese women (MO) cohort was sub-classified according to liver pathology study into normal liver (NL, $n=18$ ), simple steatosis (SS, $n=20$ ), and non-alcoholic steatohepatitis 
(NASH, $n=17$ ) (Table 1). We found no significant differences regarding age and anthropometrical measurements between the three groups studied. With regard to biochemical analysis, glucose levels were significantly increased in the SS and NASH groups compared to the NL group $(p=0.017$ and $p=0.010)$. Glycosylated hemoglobin (HbA1c) levels were also higher in SS than in NL $(p=0.039)$. Our results showed that aspartate aminotransferase (AST) and alanine aminotransferase (ALT) activity were higher in the NASH group than in the NL group $(p=0.001$ and $p \leqslant 0.001)$ and that ALT was increased in NASH compared to SS $(p=0.001)$.

Table 1. General characteristics of the studied cohort classified according to the liver pathology.

\begin{tabular}{cccc}
\hline & \multicolumn{3}{c}{ Morbidly Obese Subjects $(\boldsymbol{n}=\mathbf{5 5})$} \\
\cline { 2 - 4 } Variables & NL $(\boldsymbol{n}=\mathbf{1 8})$ & SS $(\boldsymbol{n}=\mathbf{2 0})$ & NASH $(\boldsymbol{n}=\mathbf{1 7})$ \\
\cline { 2 - 4 } & Mean \pm SD & Mean \pm SD & Mean \pm SD \\
\hline Age (years) & $48.6 \pm 10.9$ & $50.4 \pm 11.0$ & $47.8 \pm 13.0$ \\
Weight (kg) & $120.5 \pm 19.3$ & $120.4 \pm 18.1$ & $116.6 \pm 15.5$ \\
BMI (kg/m $\left.{ }^{2}\right)$ & $50.1 \pm 7.6$ & $48.8 \pm 8.5$ & $47.0 \pm 4.8$ \\
WC (cm) & $130.0 \pm 17.9$ & $129.5 \pm 12.9$ & $129.4 \pm 12.0$ \\
Glucose (mg/dL) & $94.2 \pm 22.6$ & $133.9 \pm 50.6^{*}$ & $138.7 \pm 49.1 *$ \\
Insulin (mUI/L) & $12.1 \pm 7.8$ & $18.6 \pm 12.3$ & $20.1 \pm 16.4$ \\
HbA1c (\%) & $5.2 \pm 0.9$ & $6.5 \pm 1.7 *$ & $6.3 \pm 1.6$ \\
HOMA2-IR & $1.6 \pm 0.9$ & $2.8 \pm 1.4$ & $2.8 \pm 2.1$ \\
HDL-C (mg/dL) & $44.5 \pm 9.8$ & $36.8 \pm 11.3$ & $37.1 \pm 5.9$ \\
LDL-C (mg/dL) & $99.0 \pm 27.3$ & $100.9 \pm 29.3$ & $104.4 \pm 31.2$ \\
Total cholesterol (mg/dL) & $173.03 \pm 35.53$ & $169.55 \pm 34.04$ & $174.81 \pm 33.66$ \\
Triglycerides (mg/dL) & $136.5 \pm 58.4$ & $193.1 \pm 128.6$ & $174.0 \pm 81.1$ \\
AST (U/L) & $23.5 \pm 12.3$ & $40.2 \pm 33.9$ & $64.9 \pm 35.8^{*}$ \\
ALT (U/L) & $22.1 \pm 8.5$ & $37.6 \pm 22.9$ & $67.0 \pm 33.4^{*}$ *\# \\
GGT (U/L) & $26.6 \pm 23.5$ & $27.6 \pm 14.8$ & $53.7 \pm 59.5$ \\
ALP (U/L) & $61.9 \pm 12.4$ & $74.1 \pm 20.3$ & $79.9 \pm 29.7$ \\
\hline
\end{tabular}

ALP: alkaline phosphatase; ALT: alanine aminotransferase; AST: aspartate aminotransferase; BMI: body mass index; GGT: gamma-glutamyltransferase; HbA1c: glycosylated hemoglobin; HDL-C: high density lipoprotein cholesterol; HOMA2-IR: homeostatic model assessment 2-insulin resistance; LDL-C: low density lipoprotein cholesterol; NASH: morbidly obese subjects with steatohepatitis; NL: morbidly obese subjects with normal liver; SS: morbidly obese subjects with simple steatosis; WC: waist circumference. One-way ANOVA with post-hoc Tukey test was used to compare variables between groups. * indicates statistically significant differences respect NL group $(p<0.05)$; \# indicates statistically significant differences respect SS group $(p<0.05)$. Data are expressed as mean $\pm \mathrm{SD}$.

\subsection{Determination of Patatin-Like Phospholipase Domain-Containing Protein 3 (PNPLA3) Liver Expression}

We analyzed PNPLA3 liver expression in MO women in relation to the presence of NAFLD. The results showed that PNPLA3 expression was a significant $72 \%$ greater in MO NAFLD women than in MO women with NL (MO NAFLD: $3.6 \pm 2.2$ and MO NL: $2.1 \pm 0.8, p=0.001$ ). Furthermore, when we classified the MO cohort into NL, SS, and NASH, we observed that the expression of PNPLA3 was significantly higher in SS than in NL ( $p=0.006$, Figure 1A). There were no differences between NL or SS and NASH ( $p=0.380$ and $p=0.170$, respectively). It is important to note that, in our work, any patient with steatohepatitis had fibrosis in the liver histology, so we could not perform correlations between fibrosis staging and PNPLA3 liver expression.

In addition, in order to explore the increased expression of PNPLA3 in simple steatosis, we classified the SS group into grades: mild $(n=9)$, moderate $(n=5)$, or severe SS $(n=6)$. We found that the expression of PNPLA3 was significantly increased in the severe group compared to the mild SS group $(p=0.020$, Figure $1 \mathrm{~B})$. 
(A)

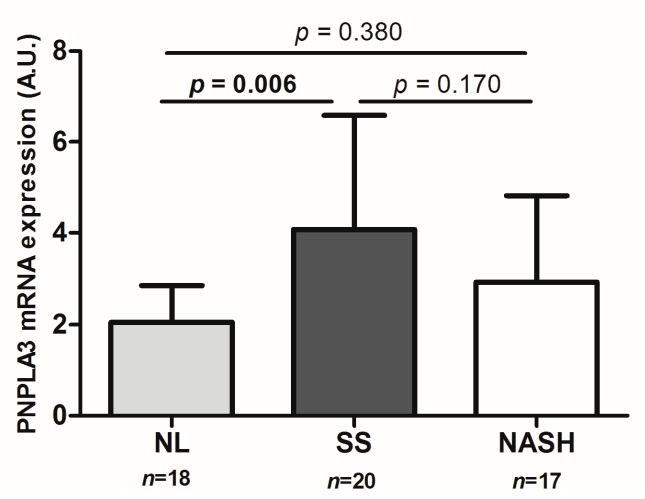

(B)

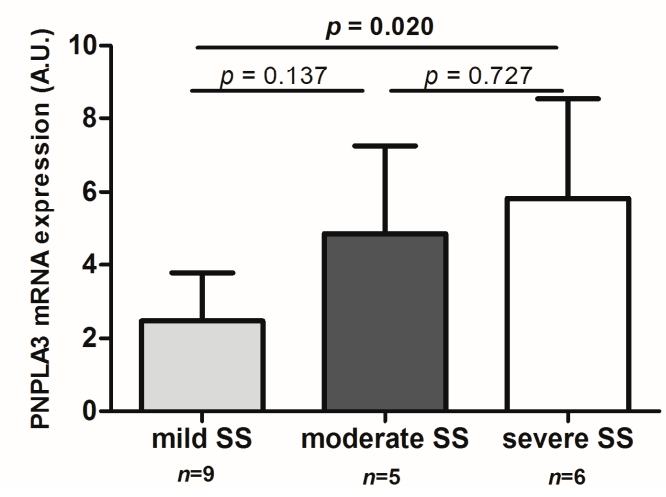

Figure 1. Hepatic expression of PNPLA3 gene in morbidly obese women according to the liver histopathology (A) and subclassifying the SS group into: mild, moderate, or severe SS (B). A.U.: arbitrary units; NASH: morbidly obese women with steatohepatitis; NL: morbidly obese women with a normal liver; SS: morbidly obese women with simple steatosis. ANOVA test was used to determinate differences between groups. $p<0.05$ are considered statistically significant.

2.3. Correlations between the Expression of PNPLA3 and Biochemical Variables, Histopathological Parameters and Genes Involved in Lipid Metabolism and Inflammation in Liver from Morbidly Obese Subjects

When we analyzed the associations between PNPLA3 expression and parameters related to glucose metabolism and lipid profile, we observed a direct correlation between circulating levels of triglycerides and PNPLA3 expression in the whole study cohort $(r=0.272, p=0.046)$.

Regarding histopathological features, we only found a direct association between PNPLA3 expression and degree of steatosis in the total MO group $(r=0.441, p=0.001)$.

In order to clarify whether PNPLA3 was associated with hepatic lipid metabolism, we studied the correlation between PNPLA3 expression and lipid metabolism related genes in liver from the $\mathrm{MO}$ cohort. In the lipogenic and fatty acid oxidation pathways, hepatic liver X receptor $(L X R \alpha)$ and peroxisome-proliferator-activated receptor $\alpha(P P A R \alpha)$ expression correlated directly with PNPLA3 expression in the total morbidly obese group $(r=0.671, p=0.008$ and $r=0.640, p=0.008$; Table 2). We also showed a positive association between PNPLA3 and both the transcription factor sterol regulatory element binding protein $2(S R E B P 2)(r=0.412, p=0.032)$ and lipocalin $2(L C N 2)(r=0.570$, $p=0.032)$ in the whole population.

Interestingly, when we analyzed the relationship between the expression of these genes in the SS subgroup, we observed that both $L X R \alpha$ and PPAR $\alpha$ correlations were stronger ( $L X R \alpha: r=0.806$, $p=0.016 ;$ PPAR $\alpha: r=0.796, p=0.024)$.

\section{4. rs738409 Genotype Distribution in Morbidly Obese Subjects}

The distribution of the studied genetic polymorphism is shown in Table 3, as are comparisons between NL, SS, and NASH patients. The G allele was more frequent $(66.6 \%)$ than the C allele $(33.3 \%)$ in the whole population. No individuals were homozygous for the $\mathrm{C}$ allele. The genotype frequencies of the rs738409 polymorphism showed significant variations between NL, SS, and NASH patients $(p=0.021)$. In addition, the GG genotype was correlated with the presence of NASH $(r=0.382$, $p<0.001)$. However, the allele frequencies did not show statistically significant differences $(p=0.145)$. Regarding clinical and biochemical variables, the GG genotype was only associated with increased body mass index (BMI) $(r=0.300, p=0.032)$. There was no association between PNPLA3 genetic variant and its hepatic expression $(p=0.478)$. 
Table 2. Correlations between PNPLA3 expression and genes related to de novo lipogenesis, FA oxidation, FA transport and uptake, inflammation, adipocytokines, and cholesterol metabolism in livers from MO women and those sub-classified as SS in the MO cohort.

\begin{tabular}{|c|c|c|c|c|}
\hline \multirow{2}{*}{ Variables } & \multicolumn{2}{|c|}{ MO PNPLA3 $(n=55)$} & \multicolumn{2}{|c|}{ SS PNPLA3 $(n=20)$} \\
\hline & $r$ & $p$-Value * & $r$ & $p$-Value * \\
\hline \multicolumn{5}{|c|}{ De Novo Lipogenesis } \\
\hline SREBP1c & -0.016 & 0.920 & 0.130 & 0.906 \\
\hline$L x R \alpha$ & 0.671 & 0.008 & 0.806 & 0.016 \\
\hline ACC1 & -0.025 & 0.920 & 0.090 & 0.906 \\
\hline FAS & -0.021 & 0.920 & 0.114 & 0.906 \\
\hline \multicolumn{5}{|c|}{ Fatty Acid Oxidation } \\
\hline PPAR $\alpha$ & 0.640 & 0.008 & 0.796 & 0.024 \\
\hline$C P T 1 \alpha$ & 0.134 & 0.576 & -0.233 & 0.906 \\
\hline CROT & 0.200 & 0.466 & 0.098 & 0.906 \\
\hline \multicolumn{5}{|c|}{ Cholesterol Metabolism } \\
\hline$A B C A 1$ & 0.016 & 0.920 & -0.189 & 0.906 \\
\hline SREBP2 & 0.412 & 0.032 & 0.361 & 0.784 \\
\hline \multicolumn{5}{|c|}{ Transport and Uptake FA } \\
\hline FABP4 & -0.371 & 0.285 & 0.464 & 0.784 \\
\hline ABCG1 & 0.099 & 0.713 & -0.074 & 0.906 \\
\hline \multicolumn{5}{|c|}{ Inflammation } \\
\hline IL6 & -0.379 & 0.285 & -0.012 & 0.980 \\
\hline$T N F \alpha$ & 0.227 & 0.576 & 0.089 & 0.906 \\
\hline LCN2 & 0.570 & 0.032 & 0.466 & 0.784 \\
\hline \multicolumn{5}{|c|}{ Adipokines } \\
\hline RESISTIN & 0.209 & 0.576 & 0.124 & 0.906 \\
\hline ADIPOR2 & -0.245 & 0.576 & 0.491 & 0.784 \\
\hline
\end{tabular}

ABCA1: ATP-binding cassette transporter A1; ABCG1: ATP-binding cassette transporter G1; ADIPOR2: adiponectin receptor; ACC1: acetyl-coenzyme A carboxylase 1; CROT: carnitine O-octanoyltransferase ; FA: fatty acid; FABP4: fatty acid binding protein 4; FAS: fatty acid synthase; IL6: interleukin 6; LCN2: lipocalin 2; $\mathrm{LXR} \alpha$ : liver X receptor; MO: morbidly obese women; PPAR $\alpha$ : peroxisome-proliferator-activated receptor $\alpha$; SREBP1c: sterol regulatory element binding protein 1c; SREBP2: sterol regulatory element binding protein 2; SS: simple steatosis; TNF $\alpha$ : tumor necrosis factor. Bold numbers indicate statistically significant correlations ( $p$-value $<0.05) .{ }^{*} p$-Value adjusted by the Benjamini-Hochberg method [24].

Table 3. The distribution of rs738409 polymorphism in morbidly obese women according to liver histology.

\begin{tabular}{ccccc}
\hline \multirow{2}{*}{ Groups } & \multicolumn{2}{c}{ Genotype, $\boldsymbol{n}(\mathbf{\%})$} & \multicolumn{2}{c}{ Allele, $\boldsymbol{n}(\mathbf{\%})$} \\
\cline { 2 - 5 } & CG & GG & C & G \\
\hline NL $(n=16)$ & $12(75)$ & $4(25)$ & $12(37.5)$ & $20(62.5)$ \\
SS $(n=18)$ & $15(83.3)$ & $3(16.6)$ & $15(41.7)$ & $21(58.3)$ \\
NASH $(n=17)$ & $7(41.2)$ & $10(58.8)$ & $7(20.6)$ & $27(79.4)$ \\
\hline
\end{tabular}

CG: individuals carrying the genotype (CG); GG: individuals carrying the genotype (GG); C: Allele C; G: Allele G; NASH: morbidly obese subjects with steatohepatitis; NL: morbidly obese subjects with normal liver; SS: morbidly obese subjects with simple steatosis.

\section{Discussion}

In an own previous work, we demonstrated a downregulation of the lipogenic pathway related to the severity of steatosis in a cohort of women with morbid obesity [25]. As PNPLA3 seems to be related with the accumulation of hepatic TG, in the present study, we examined the relationship between the liver expression levels of PNPLA3, the key lipid metabolism-related genes expression, 
and the clinicopathological factors in a cohort of morbidly obese women with NAFLD. In our study, $36 \%$ and $31 \%$ of morbidly obese women were diagnosed with SS and NASH, respectively, using the diagnostic gold standard liver biopsy. Our findings show that PNPLA3 liver expression was increased in morbidly obese women with NAFLD. It is important to note that we have demonstrated a clear relationship between PNPLA3 and the degrees of SS, suggesting a direct correlation between PNPLA3 and the severity of steatosis.

Nowadays, more than 50 studies on the genotyping of PNPLA3 have confirmed the association between the $148 \mathrm{M}$ variant and the full range of NAFLD, including simple steatosis, steatohepatitis, cirrhosis, and hepatocellular carcinoma. PNPLA3 148M has been shown to be related to an increased risk of NAFLD across multiple ethnic groups [26-34]. The aim of the present work was to compare the hepatic expression of PNPLA3 in a cohort of morbidly obese women presenting a normal liver or NAFLD. We showed that the hepatic expression of PNPLA3 in morbidly obese women with NAFLD was higher than in MO women with NL. Consistent with our work, Kotronen et al. [8] described a direct correlation between PNPLA3 liver expression and liver fat content measured by magnetic resonance. It is important to note that our study confirms this finding in biopsy-proven NAFLD. Regarding steatosis degree, recent studies observed that a variant of this protein has an association with moderate-to-severe steatosis [35,36]. Although these studies analyzed only a variant of PNPLA3, not its liver expression, their results are in agreement with ours. A recent interesting work by Donati et al. [37] has demonstrated that PNPLA3 rs2294918 E434K diminished PNPLA3 expression and protein levels, lessening the effect of the rs738409 polymorphism on the predisposition to steatosis liver injury. Moreover, the authors suggested that this PNPLA3 variant had a codominant negative effect on TG mobilization from lipid droplets. Regarding non-alcoholic steatohepatitis, a DNA microarray study in human liver revealed an upregulation of PNPLA3 in NASH vs. healthy controls [38]. Nevertheless, Kitamoto et al. [39] described lower PNPLA3 mRNA levels in the liver of patients with an advanced grade of NAFLD (with fibrosis) compared with those with mild NAFLD. However, we were not able to reproduce any of these findings. Perhaps the differences in the groups studied in these works regarding age, gender, BMI, or race can explain these discrepancies.

Because PNPLA3 has previously been reported to influence lipid metabolism in animal models and in in vitro studies [40,41], we evaluated the interplay of PNPLA3 liver expression with the expression of the main lipid metabolism-related genes. In the current first human study in this sense, PNPLA3 expression positively correlated with $L X R \alpha, P P A R \alpha$, and SREBP2 liver expression. All these proteins are transcription factors that relate to response elements found in a various genes that are associated with lipid turnover including their own genes [42]. Specifically, LXR $\alpha$ belong to the nuclear hormone receptor superfamily of ligand-activated transcription factors as SREBP1 which, in liver, serve as lipid sensors and regulate the expression of main genes which modulate the cholesterol and FA metabolism [43]. Regarding NAFLD, interaction between LXR and SREBP1 is a crucial step in the molecular cascade of events characterizing steatogenesis [44]. In this regard, Huang et al. [41] determined that the overexpression of the three SREBP family members (SREBP1a, 1c, and 2) increases liver PNPLA3 expression in mice. They also found that PNPLA3 expression was regulated by SREBP1c and $L X R \alpha$. Similar results were described by Dubuquoy et al. [45], who showed that, in the mouse liver, PNPLA3 gene expression was under the direct transcriptional control of SREBP1c in response to insulin. However, at variance with murine studies, we were not able to find any association with SREBP1, one of the key genes related to de novo lipogenesis. Moreover, Mancina et al. [34] conducted a study to evaluate the contribution of de novo lipogenesis to liver fat accumulation in the PNPLA3 I148M genetic variant of NAFLD. They showed a dissociation between hepatic de novo lipogenesis and liver fat content due to the PNPLA3 148M allele, suggesting that increased de novo lipogenesis is not a main feature in all subjects with steatosis. However, these authors have not studied the hepatic expression of PNPLA3. Regarding the positive relationship between PPAR $\alpha$ and PNPLA3, it is known that PPAR $\alpha$ seems to control the expression of genes regulating peroxisomal/mitochondrial $\beta$-oxidation [46]. In this context, perhaps the induction of fatty acid catabolism might act as a defense 
mechanism, preventing hepatocellular fat accumulation [47]; in other words, it might represent an inefficient physiological response to counteract steatosis by promoting the $\beta$-oxidation of fatty acids in the hepatocytes. In our study, the association between PNPLA3 and SREBP2 may suggest a novel association with cholesterol metabolism in humans. Currently, experimental and human evidence has related to altered hepatic cholesterol metabolism and free cholesterol accumulation to the pathogenesis of steatosis and liver damage [48]. Specifically, Min et al. [49] have demonstrated dysregulated cholesterol metabolism in NAFLD, which may contribute to disease severity through activation of SREBP2 and 3-Hydroxy-3-Methylglutaryl-CoA Reductase (HMGCR).

In the present work, we observed an interesting association between liver PNPLA3 expression and the liver expression of LCN2 in the severely obese women group, which has not been previously described. In one of our previous studies, we described increased liver LCN2 expression in NAFLD, and this expression positively correlated with SS [50]. Additionally, in this work, an increased regulation of LCN2 expression was detected in in vitro experiments with HepG2 cells under harmful conditions. Perhaps, as some authors have suggested, LCN2 is a protective molecule [51] —in this case, against the development of NAFLD.

Moreover, we did not find any relationship between PNPLA3 liver expression and other adipocytokines studied, probably because the molecular function of PNPLA3 is related to cellular lipid accumulation in the liver more than with inflammation [52]. Unexpectedly, we did not find any relationship with the expression of genes related to transport and the uptake of fatty acids. Perhaps this mechanism of liver accumulation of fatty acids has a lower contribution in humans, as we and other authors have previously shown $[25,38]$.

Finally, to explore the effect of the PNPLA3 genetic variant with a potential impact on NAFLD, we determined the relationship between the rs738409 polymorphism in the PNPLA3 gene and the severity of disease. In this sense, we found that the GG genotype, encoding I148M, was directly correlated with the presence of NASH. Our results are similar to recent studies that showed a relationship between this genetic variant and the severity of NAFLD [12,13,15,53]. Consistent with our results, Kotronen et al. [8] observed that there were no differences in the hepatic PNPLA3 mRNA expression between different PNPLA3 genotype carriers.

We should point out the following drawbacks of our study. The main limitation of this work is an adjusted sample size and the lack of evaluation of protein expression. Additionally, the study is cross-sectional. We could not prove a causal link between PNPLA3 expression and NALFD development. However, our study cohort of morbidly obese women has revealed clear relationships between the expression of PNPLA3 and NAFLD, without the interference of gender or age. Thus, our findings cannot be extrapolated to men or other obesity groups such as normal-weight or over-weight women.

\section{Materials and Methods}

\subsection{Subjects}

The study was approved by the ethics committee of the Hospital Joan XXIII (23c/2015, Tarragona, Spain), and all subjects gave written informed consent. We included 55 Caucasian MO women $\left(\mathrm{BMI}>40 \mathrm{~kg} / \mathrm{m}^{2}\right)$. Liver biopsies were obtained during planned laparoscopic bariatric surgery and were performed for clinical indications.

The diagnosis of NAFLD was made using the following criteria: (1) liver pathology; (2) an intake of less than $10 \mathrm{~g}$ of ethanol/day; and (3) appropriate exclusion of other liver diseases.

The body weight of all women had not fluctuated more than $2 \%$ for at least 3 months prior to bariatric surgery. The exclusion criteria were: (1) concurrent use of medications known to produce hepatic steatosis; (2) patients using hypolipemiant treatment; (3) diabetic subjects who were receiving insulin or on medication likely to influence endogenous insulin levels; (4) menopausal or post-menopausal women; (5) women undergoing contraceptive treatment and subjects receiving 
contraceptive treatment; (6) patients who had an acute illness, current evidence of acute or chronic inflammatory or infectious diseases, or malignant diseases.

\subsection{Liver Pathology}

Liver samples were processed by two experienced hepatopathologists using methods previously described [54,55]. Simple steatosis (SS) was graded as follows: Grade 1 or mild SS: more than $5 \%$ and less than $33 \%$ of hepatocytes affected; Grade 2 or moderate SS: $33 \%$ to $66 \%$ of hepatocytes affected; or Grade 3 or severe SS: more than $66 \%$ of hepatocytes affected. Moreover, the minimum criteria for the steatohepatitis diagnosis included the presence of either ballooning cells and lobular inflammation or perisinusoidal/pericellular fibrosis in zone 3 of the hepatic acinus.

According to liver pathology, women were sub-classified into: (1) normal liver (NL) histology $(n=18)$; (2) simple steatosis (SS) (micro/macrovesicular steatosis without inflammation or fibrosis, $n=20$ ); (3) non-alcoholic steatohepatitis (NASH) (Brunt Grades 1-3, $n=17$ ).

\subsection{Biochemical Analyses}

Each of our patients was evaluated with a complete physical, anthropometrical, and biochemical assessment. BMI was calculated as body weight divided by height squared $\left(\mathrm{kg} / \mathrm{m}^{2}\right)$. Fasting glucose, insulin, HbA1c, HDL-C, LDL-C, TG, and transaminases were measured using a conventional automated analyzer after overnight fasting. Insulin resistance was calculated using HOMA2-IR [56].

\subsection{RNA Isolation and Real-Time PCR}

Liver samples were preserved in RNAlater (Sigma, Barcelona, Spain) for $24 \mathrm{~h}$ at $4{ }^{\circ} \mathrm{C}$ and then stored at $-80^{\circ} \mathrm{C}$. Total RNA was extracted by using an RNeasy mini kit (Qiagen, Barcelona, Spain). And was reverse transcribed by the High Capacity RNA-to-cDNA Kit (Applied Biosystems, Madrid, Spain). Real-time quantitative PCR was carried out with the TaqMan Assay predesigned by Applied Biosystems for the detection of PNPLA3 (Hs00228747_m1), ABCA1 (Hs01059118_m1), ABCG1 (Hs00245154_m1), ADIPOR2 (Hs00226105_m1), ACC1 (Hs00167385_m1), CROT (Hs00221733_m1), FABP4 (Hs00609791_m1), FAS (Hs00188012_m1), IL6 (Hs00985639_m1), LCN2 (Hs00194353_m1), LXR (Hs00173195_m1), PPAR (Hs00947538_m1), RESISTIN (Hs00220767_m1), SREBP1c (Hs01088691_m1), SREBP2 (Hs01081784_m1), TNF (Hs99999043_m1), and 18S ribosomal RNA (4352930E), which was used as the housekeeping gene. All reactions were performed in duplicate using the 7900HT Fast Real-Time PCR systems (Applied Biosystems).

\subsection{Genotyping}

Subjects were genotyped for the rs738409 polymorphism using the TaqMan $5^{\prime}$ allelic discrimination assay (TaqMan SNP Genotyping Assay C 7241 10, Applied Biosystems,). Amplifications were carried out using the 7900HT Sequencing Detection System for continuous fluorescence monitoring.

\subsection{Statistical Analysis}

We used the SPSS/PC+ statistical package for Windows (version 22.0; SPSS, Chicago, IL, USA). One-way ANOVA with a post-hoc Tukey test was carried out to determine differences between groups. The correlations between variables was analyzed using Pearson's method (parametric variables) and Spearman's test (non-parametric variables). Allele and genotype frequencies were evaluated with the $\chi$-squared test. $p$-Values $<0.05$ were considered to be statistically significant.

\section{Conclusions}

The main results of our study show that liver PNPLA3 expression is increased in NAFLD patients and is particularly associated to severity of steatosis. Moreover, PNPLA3 expression is correlated with 
the expression of main cholesterol and hepatic lipid metabolism-related genes. Further human studies are required to confirm these associations.

Acknowledgments: This study was supported by the Fondo de Investigación Sanitaria and Fondo Europeo de Desarrollo Regional (FEDER, grant number PI13/00468, to Teresa Auguet), by funds from Agència de Gestió d'Ajuts Universitaris de Recerca (AGAUR 2009 SGR 959 to Cristóbal Richart) and the Grup de Recerca en Medicina Aplicada-Universitat Rovira Virgili (2015 PFR-URV-B2-72 to Cristóbal Richart), and by the Fundación Biociencia.

Author Contributions: Gemma Aragonès and Teresa Auguet participated in the design of the study and in the analysis and interpretation of data and were involved in the drafting of the manuscript. Teresa Auguet reviewed/edited the manuscript. Sandra Armengol, Alba Berlanga, Esther Guiu-Jurado, and Carmen Aguilar carried out the experimental work. Salomé Martinez and Joan Josep Sirvent are the pathologists. Maikel Daniel Ruiz, Fátima Sabench, Mercé Hernández, José Antonio Porras, and Daniel Del Castillo made substantial contributions to the conception and design of the study, and to the acquisition of the samples. Cristóbal Richart revised the draft and gave the final approval for publication. The authors have all seen the final version.

Conflicts of Interest: The authors declare no conflict of interest.

\section{Abbreviations}

\begin{tabular}{ll} 
ABCA1 & ATP-binding cassette transporter A1 \\
ABCG1 & ATP-binding cassette transporter G1 \\
ADIPOR & adiponectin receptor \\
ACC1 & acetyl-coenzyme A carboxylase 1 \\
ALT & alanine aminotransaminase \\
AST & aspartate aminotransaminase \\
ALP & alkaline phosphatase \\
BMI & body mass index \\
CROT & carnitine O-octanoyltransferase \\
FABP4 & fatty acid binding protein 4 \\
FAS & fatty acid synthase \\
18S & 18S ribosomal RNA \\
GGT & $\gamma$-glutamyl transferase \\
HbA1c & glycosylated hemoglobin \\
HDL-C & high density lipoprotein \\
HOMA2-IR & homeostasis model assessment of insulin resistance \\
IL6 & interleukin 6 \\
LDL-C & low density lipoprotein \\
LCN2 & lipocalin 2 \\
LXR $\alpha$ & liver X receptor \\
MO & morbidly obese \\
NAFLD & non-alcoholic fatty liver disease \\
NASH & non-alcoholic steatosis \\
NL & normal liver \\
PPAR $\alpha$ & peroxisome-proliferator-activated receptor $\alpha$ \\
SREBP1c & sterol regulatory element binding protein 1c \\
SREBP2 & sterol regulatory element binding protein 2 \\
SS & simple steatosis \\
TG & triglycerides \\
TNF $\alpha$ & tumor necrosis factor \\
WC & waist circumference \\
& \\
\hline &
\end{tabular}




\section{References}

1. Lonardo, A.; Bellentani, S.; Argo, C.K.; Ballestri, S.; Byrne, C.D.; Caldwell, S.H.; Cortez-Pinto, H.; Grieco, A.; Machado, M.V.; Miele, L.; et al. Epidemiological modifiers of non-alcoholic fatty liver disease: Focus on high-risk groups. Dig. Liver Dis. 2015, 47, 997-1006. [CrossRef]

2. Farrell, G.C.; Larter, C.Z. Nonalcoholic fatty liver disease: From steatosis to cirrhosis. Hepatology 2006, 43, S99-S112. [CrossRef]

3. Younossi, Z.M.; Koenig, A.B.; Abdelatif, D.; Fazel, Y.; Henry, L.; Wymer, M. Global epidemiology of non-alcoholic fatty liver disease-meta-analytic assessment of prevalence, incidence and outcomes. Hepatology 2015. [CrossRef]

4. Ballestri, S.; Romagnoli, D.; Nascimbeni, F.; Francica, G.; Lonardo, A. Role of ultrasound in the diagnosis and treatment of nonalcoholic fatty liver disease and its complications. Expert Rev. Gastroenterol. Hepatol. 2015, 9, 603-627. [CrossRef]

5. Buzzetti, E.; Pinzani, M.; Tsochatzis, E.A. The multiple-hit pathogenesis of non-alcoholic fatty liver disease (NAFLD). Metabolism 2016, 1-11. [CrossRef]

6. Vernon, G.; Baranova, A.; Younossi, Z.M. Systematic review: The epidemiology and natural history of non-alcoholic fatty liver disease and non-alcoholic steatohepatitis in adults. Aliment. Pharmacol. Ther. 2011, 34, 274-285. [CrossRef]

7. Daly, A.K.; Ballestri, S.; Carulli, L.; Loria, P.; Day, C.P. Genetic determinants of susceptibility and severity in nonalcoholic fatty liver disease. Expert Rev. Gastroenterol. Hepatol. 2011, 5, 253-263. [CrossRef]

8. Kotronen, A.; Johansson, L.E.; Johansson, L.M.; Roos, C.; Westerbacka, J.; Hamsten, A.; Bergholm, R.; Arkkila, P.; Arola, J.; Kiviluoto, T.; et al. A common variant in PNPLA3, which encodes adiponutrin, is associated with liver fat content in humans. Diabetologia 2009, 52, 1056-1060. [CrossRef]

9. Sookoian, S.; Pirola, C.J. PNPLA3, the triacylglycerol synthesis/hydrolysis/storage dilemma, and nonalcoholic fatty liver disease. World J. Gastroenterol. 2012, 18, 6018-6026. [CrossRef]

10. Jenkins, C.M.; Mancuso, D.J.; Yan, W.; Sims, H.F.; Gibson, B.; Gross, R.W. Identification, cloning, expression, and purification of three novel human calcium-independent phospholipase A2 family members possessing triacylglycerol lipase and acylglycerol transacylase activities. J. Biol. Chem. 2004, 279, 48968-48975. [CrossRef]

11. Lake, A.C.1.; Sun, Y.; Li, J.L.; Kim, J.E.; Johnson, J.W.; Li, D.; Revett, T.; Shih, H.H.; Liu, W.; Paulsen, J.E.; et al. Expression, regulation, and triglyceride hydrolase activity of Adiponutrin family members. J. Lipid Res. 2005, 46, 2477-2487. [CrossRef]

12. Romeo, S.; Kozlitina, J.; Xing, C.; Pertsemlidis, A.; Cox, D.; Pennacchio, L.A.; Boerwinkle, E.; Cohen, J.C.; Hobbs, H.H. Genetic variation in PNPLA3 confers susceptibility to nonalcoholic fatty liver disease. Nat. Genet. 2008, 40, 1461-1465. [CrossRef]

13. Rotman, Y.; Koh, C.; Zmuda, J.M.; Kleiner, D.E.; Liang, T.J. The association of genetic variability in patatin-like phospholipase domain-containing protein 3 (PNPLA3) with histological severity of nonalcoholic fatty liver disease. Hepatology 2010, 52, 894-903. [CrossRef]

14. Valenti, L.; Alisi, A.; Galmozzi, E.; Bartuli, A.; Del Menico, B.; Alterio, A.; Dongiovanni, P.; Fargion, S.; Nobili, V. I148M patatin-like phospholipase domain-containing 3 gene variant and severity of pediatric nonalcoholic fatty liver disease. Hepatology 2010, 52, 1274-1280. [CrossRef]

15. Sookoian, S.; Pirola, C.J. Meta-analysis of the influence of I148M variant of patatin-like phospholipase domain containing 3 gene (PNPLA3) on the susceptibility and histological severity of nonalcoholic fatty liver disease. Hepatology 2011, 53, 1883-1894. [CrossRef]

16. Krawczyk, M.; Grünhage, F.; Zimmer, V.; Lammert, F. Variant adiponutrin (PNPLA3) represents a common fibrosis risk gene: Non-invasive elastography-based study in chronic liver disease. J. Hepatol. 2011, 55, 299-306. [CrossRef]

17. Valenti, L.; Al-Serri, A.; Daly, A.K.; Galmozzi, E.; Rametta, R.; Dongiovanni, P.; Nobili, V.; Mozzi, E.; Roviaro, G.; Vanni, E.; et al. Homozygosity for the patatin-like phospholipase-3/adiponutrin I148M polymorphism influences liver fibrosis in patients with nonalcoholic fatty liver disease. Hepatology 2010, 51, 1209-1217. [CrossRef]

18. Zain, S.M.; Mohamed, R.; Mahadeva, S.; Cheah, P.L.; Rampal, S.; Basu, R.C.; Mohamed, Z. A multi-ethnic study of a PNPLA3 gene variant and its association with disease severity in non-alcoholic fatty liver disease. Hum. Genet. 2012, 131, 1145-1152. [CrossRef] [PubMed] 
19. Kovarova, M.; Königsrainer, I.; Königsrainer, A.; Machicao, F.; Häring, H.-U.; Schleicher, E.; Peter, A. The genetic variant I148M in PNPLA3 is associated with increased hepatic retinyl-palmitate storage in humans. J. Clin. Endocrinol. Metab. 2015, 100, E1568-E1574. [CrossRef] [PubMed]

20. Pirazzi, C.; Valenti, L.; Motta, B.M.; Pingitore, P.; Hedfalk, K.; Mancina, R.M.; Burza, M.A.; Indiveri, C.; Ferro, Y.; Montalcini, T.; et al. PNPLA3 has retinyl-palmitate lipase activity in human hepatic stellate cells. Hum. Mol. Genet. 2014, 23, 4077-4085. [CrossRef] [PubMed]

21. Chamoun, Z.; Vacca, F.; Parton, R.G.; Gruenberg, J. PNPLA3/adiponutrin functions in lipid droplet formation. Biol. Cell 2013, 105, 219-233. [CrossRef] [PubMed]

22. Ruhanen, H.; Perttilä, J.; Hölttä-Vuori, M.; Zhou, Y.; Yki-Järvinen, H.; Ikonen, E.; Käkelä, R.; Olkkonen, V.M. PNPLA3 mediates hepatocyte triacylglycerol remodelling. J. Lipid Res. 2014, 55, 739-746. [CrossRef] [PubMed]

23. Min, H.-K.; Sookoian, S.; Pirola, C.J.; Cheng, J.; Mirshahi, F.; Sanyal, A.J. Metabolic profiling reveals that PNPLA3 induces widespread effects on metabolism beyond triacylglycerol remodeling in Huh-7 hepatoma cells. Am. J. Physiol. Gastrointest. Liver Physiol. 2014, 307, G66-G76. [CrossRef] [PubMed]

24. Benjamini, Y.; Hochberg, Y. Controlling the false discovery rate: A practical and powerful approach to multiple testing. J. R. Stat. Soc. B 1995, 57, 289-300.

25. Auguet, T.; Berlanga, A.; Guiu-Jurado, E.; Martinez, S.; Porras, J.A.; Aragonès, G.; Sabench, F.; Hernandez, M.; Aguilar, C.; Sirvent, J.J.; et al. Altered fatty acid metabolism-related gene expression in liver from morbidly obese women with non-alcoholic fatty liver disease. Int. J. Mol. Sci. 2014, 15, 22173-22187. [CrossRef] [PubMed]

26. Lin, Y.-C.; Chang, P.-F.; Chang, M.-H.; Ni, Y.-H. Genetic variants in GCKR and PNPLA3 confer susceptibility to nonalcoholic fatty liver disease in obese individuals. Am. J. Clin. Nutr. 2014, 99, 869-874. [CrossRef] [PubMed]

27. Wu, P.; Shu, Y.; Guo, F.; Luo, H.; Zhang, G.; Tan, S. [Association between patatin-like phospholipase domain-containing protein 3 gene rs738409 polymorphism and non-alcoholic fatty liver disease susceptibility: A meta-analysis]. Zhonghua Liu Xing Bing Xue Za Zhi 2015, 36, 78-82. [PubMed]

28. Xu, R.; Tao, A.; Zhang, S.; Deng, Y.; Chen, G. Association between patatin-like phospholipase domain containing 3 gene (PNPLA3) polymorphisms and nonalcoholic fatty liver disease: A HuGE review and meta-analysis. Sci. Rep. 2015, 5, 9284. [CrossRef] [PubMed]

29. Zhang, L.; You, W.; Zhang, H.; Peng, R.; Zhu, Q.; Yao, A.; Li, X.; Zhou, Y.; Wang, X.; Pu, L.; et al. PNPLA3 polymorphisms (rs738409) and non-alcoholic fatty liver disease risk and related phenotypes: A meta-analysis. J. Gastroenterol. Hepatol. 2015, 30, 821-829. [CrossRef] [PubMed]

30. León-Mimila, P.; Vega-Badillo, J.; Gutiérrez-Vidal, R.; Villamil-Ramírez, H.; Villareal-Molina, T.; Larrieta-Carrasco, E.; López-Contreras, B.E.; Kauffer, L.R.M.; Maldonado-Pintado, D.G.; Méndez-Sánchez, N.; et al. A genetic risk score is associated with hepatic triglyceride content and non-alcoholic steatohepatitis in Mexicans with morbid obesity. Exp. Mol. Pathol. 2015, 98, 178-183. [CrossRef] [PubMed]

31. Zhang, Y.; Cai, W.; Song, J.; Miao, L.; Zhang, B.; Xu, Q.; Zhang, L.; Yao, H. Association between the PNPLA3 I148M polymorphism and non-alcoholic fatty liver disease in the Uygur and Han ethnic groups of northwestern China. PLoS ONE 2014, 9, e108381. [CrossRef] [PubMed]

32. Tai, C.-M.; Huang, C.-K.; Tu, H.-P.; Hwang, J.-C.; Chang, C.-Y.; Yu, M.-L. PNPLA3 genotype increases susceptibility of nonalcoholic steatohepatitis among obese patients with nonalcoholic fatty liver disease. Surg. Obes. Relat. Dis. 2014, 11, 888-894. [CrossRef] [PubMed]

33. Lee, S.S.; Byoun, Y.-S.; Jeong, S.-H.; Woo, B.H.; Jang, E.S.; Kim, J.-W.; Kim, H.Y. Role of the PNPLA3 I148M polymorphism in nonalcoholic fatty liver disease and fibrosis in Korea. Dig. Dis. Sci. 2014, 59, 2967-2974. [CrossRef] [PubMed]

34. Margherita Mancina, R.; Matikainen, N.; Maglio, C.; Söderlund, S.; Lundbom, N.; Hakkarainen, A.; Rametta, R.; Mozzi, E.; Fargion, S.; Valenti, L.; et al. Paradoxical dissociation between hepatic fat content and de novo lipogenesis due to PNPLA3 sequence variant. J. Clin. Endocrinol. Metab. 2015, 100, E821-E825. [CrossRef] [PubMed]

35. Shang, X.R.; Song, J.Y.; Liu, F.H.; Ma, J.; Wang, H.J. GWAS-identified common variants with nonalcoholic fatty liver disease in chinese children. J. Pediatr. Gastroenterol. Nutr. 2015, 60, 669-674. [CrossRef] [PubMed] 
36. Nobili, V.; Liccardo, D.; Bedogni, G.; Salvatori, G.; Gnani, D.; Bersani, I.; Alisi, A.; Valenti, L.; Raponi, M. Influence of dietary pattern, physical activity, and I148M PNPLA3 on steatosis severity in at-risk adolescents. Genes Nutr. 2014, 9, 392. [CrossRef] [PubMed]

37. Donati, B.; Motta, B.M.; Pingitore, P.; Meroni, M.; Pietrelli, A.; Alisi, A.; Petta, S.; Xing, C.; Dongiovanni, P.; del Menico, B.; et al. The rs2294918 E434K variant modulates patatin-like phospholipase domain-containing 3 expression and liver damage. Hepatology 2016, 63, 787-798. [CrossRef] [PubMed]

38. Arendt, B.M.; Comelli, E.M.; Ma, D.W.L.; Lou, W.; Teterina, A.; Kim, T.; Fung, S.K.; Wong, D.K.H.; McGilvray, I.; Fischer, S.E.; et al. Altered hepatic gene expression in nonalcoholic fatty liver disease is associated with lower hepatic n-3 and n-6 polyunsaturated fatty acids. Hepatology 2015, 61, 1565-1578. [CrossRef]

39. Kitamoto, T.; Kitamoto, A.; Ogawa, Y.; Honda, Y.; Imajo, K.; Saito, S.; Yoneda, M.; Nakamura, T.; Nakajima, A.; Hotta, K. Targeted-bisulfite sequence analysis of the methylation of CpG islands in genes encoding PNPLA3, SAMM50, and PARVB of patients with non-alcoholic fatty liver disease. J. Hepatol. 2015, 63, 494-502. [CrossRef] [PubMed]

40. Hao, L.; Ito, K.; Huang, K.H.; Sae-tan, S.; Lambert, J.D.; Ross, A.C. Shifts in dietary carbohydrate-lipid exposure regulate expression of the non-alcoholic fatty liver disease-associated gene PNPLA3/adiponutrin in mouse liver and HepG2 human liver cells. Metabolism 2014, 63, 1352-1362. [CrossRef] [PubMed]

41. Huang, Y.; He, S.; Li, J.Z.; Seo, Y.-K.; Osborne, T.F.; Cohen, J.C.; Hobbs, H.H. A feed-forward loop amplifies nutritional regulation of PNPLA3. Proc. Natl. Acad. Sci. USA 2010, 107, 7892-7897. [CrossRef] [PubMed]

42. Bechmann, L.P.; Hannivoort, R.A.; Gerken, G.; Hotamisligil, G.S.; Trauner, M.; Canbay, A. The interaction of hepatic lipid and glucose metabolism in liver diseases. J. Hepatol. 2012, 56, 952-964. [CrossRef] [PubMed]

43. Zhang, X.; Liu, J.; Su, W.; Wu, J.; Wang, C.; Kong, X.; Gustafsson, J.-Å.; Ding, J.; Ma, X.; Guan, Y. Liver X receptor activation increases hepatic fatty acid desaturation by the induction of SCD1 expression through an LXR $\alpha$-SREBP1c-dependent mechanism. J. Diabetes 2014, 6, 212-220. [CrossRef] [PubMed]

44. Ballestri, S.; Nascimbeni, F.; Romagnoli, D.; Baldelli, E.; Lonardo, A. The role of nuclear receptors in the pathophysiology, natural course, and drug treatment of NAFLD in humans. Adv. Ther. 2016, 33, 291-319. [CrossRef] [PubMed]

45. Dubuquoy, C.; Robichon, C.; Lasnier, F.; Langlois, C.; Dugail, I.; Foufelle, F.; Girard, J.; Burnol, A.F.; Postic, C.; Moldes, M. Distinct regulation of adiponutrin/PNPLA3 gene expression by the transcription factors ChREBP and SREBP1c in mouse and human hepatocytes. J. Hepatol. 2011, 55, 145-153. [CrossRef] [PubMed]

46. Pawlak, M.; Lefebvre, P.; Staels, B. Molecular mechanism of PPAR $\alpha$ action and its impact on lipid metabolism, inflammation and fibrosis in non-alcoholic fatty liver disease. J. Hepatol. 2015, 62, 720-733. [CrossRef] [PubMed]

47. Dongiovanni, P.; Valenti, L. Peroxisome proliferator-activated receptor genetic polymorphisms and nonalcoholic Fatty liver disease: Any role in disease susceptibility? PPAR Res. 2013, 2013, 452061. [CrossRef] [PubMed]

48. Musso, G.; Gambino, R.; Cassader, M. Cholesterol metabolism and the pathogenesis of non-alcoholic steatohepatitis. Prog. Lipid Res. 2013, 52, 175-191. [CrossRef] [PubMed]

49. Min, H.-K.; Kapoor, A.; Fuchs, M.; Mirshahi, F.; Zhou, H.; Maher, J.; Kellum, J.; Warnick, R.; Contos, M.J.; Sanyal, A.J. Increased hepatic synthesis and dysregulation of cholesterol metabolism is associated with the severity of nonalcoholic fatty liver disease. Cell Metab. 2012, 15, 665-674. [CrossRef] [PubMed]

50. Auguet, T.; Terra, X.; Quintero, Y.; Martínez, S.; Manresa, N.; Porras, J.A.; Aguilar, C.; Orellana-Gavaldà, J.M.; Hernández, M.; Sabench, F.; et al. Liver lipocalin 2 expression in severely obese women with non alcoholic fatty liver disease. Exp. Clin. Endocrinol. Diabetes 2013, 121, 119-124. [CrossRef] [PubMed]

51. Lee, E.-K.; Kim, H.-J.; Lee, K.-J.; Lee, H.-J.; Lee, J.-S.; Kim, D.-G.; Hong, S.-W.; Yoon, Y.; Kim, J.-S. Inhibition of the proliferation and invasion of hepatocellular carcinoma cells by lipocalin 2 through blockade of JNK and PI3K/Akt signaling. Int. J. Oncol. 2011, 38, 325-333. [CrossRef] [PubMed]

52. Kumari, M.; Schoiswohl, G.; Chitraju, C.; Paar, M.; Rangrez, A.Y.; Wongsiriroj, N.; Nagy, H.M.; Pavlina, T.; Scott, S.A.; Knittelfelder, O.; et al. Adiponutrin functions as a nutritionally regulated lysophosphatidic acid acyltransferase. Cell Metab. 2012, 15, 691-702. [CrossRef] [PubMed]

53. Chen, L.Z.; Xin, Y.N.; Geng, N.; Jiang, M.; Zhang, D.D.; Xuan, S.Y. PNPLA3 I148M variant in nonalcoholic fatty liver disease: Demographic and ethnic characteristics and the role of the variant in nonalcoholic fatty liver fibrosis. World J. Gastroenterol. 2015, 21, 794-802. [PubMed] 
54. Kleiner, D.E.; Brunt, E.M.; Van Natta, M.; Behling, C.; Contos, M.J.; Cummings, O.W.; Ferrell, L.D.; Liu, Y.-C.; Torbenson, M.S.; Unalp-Arida, A.; et al. Design and validation of a histological scoring system for nonalcoholic fatty liver disease. Hepatology 2005, 41, 1313-1321. [CrossRef] [PubMed]

55. Brunt, E.M.; Janney, C.G.; Di Bisceglie, A.M.; Neuschwander-Tetri, B.A.; Bacon, B.R. Nonalcoholic steatohepatitis: A proposal for grading and staging the histological lesions. Am. J. Gastroenterol. 1999, 94, 2467-2474. [CrossRef] [PubMed]

56. Terra, X.; Quintero, Y.; Auguet, T.; Porras, J.A.; Hernández, M.; Sabench, F.; Aguilar, C.; Luna, A.M.; Del Castillo, D.; Richart, C. FABP 4 is associated with inflammatory markers and metabolic syndrome in morbidly obese women. Eur. J. Endocrinol. 2011, 164, 539-547. [CrossRef] [PubMed]

(C) 2016 by the authors; licensee MDPI, Basel, Switzerland. This article is an open access article distributed under the terms and conditions of the Creative Commons Attribution (CC-BY) license (http://creativecommons.org/licenses/by/4.0/). 